\title{
Existence and multiplicity results for some generalized Hammerstein equations with a parameter
}

\author{
Lucía López-Somoza' and Feliz Minhós ${ }^{2,3 *}$
}

\section{"Correspondence:}

fminhos@uevora.pt

2 Departamento de Matemática,

Escola de Ciências e Tecnologia,

Universidade de Évora, Évora,

Portugal

${ }^{3}$ Centro de Investigação em Matemática e Aplicações (CIMA)

Instituto de Investigação e

Formação Avançada, Universidade

de Évora, Évora, Portugal

Full list of author information is

available at the end of the article

\section{Abstract}

This paper considers the existence and multiplicity of fixed points for the integral operator

$$
\mathcal{T} u(t)=\lambda \int_{0}^{T} k(t, s) f\left(s, u(s), u^{\prime}(s), \ldots, u^{(m)}(s)\right) \mathrm{d} s, \quad t \in[0, T] \equiv l,
$$

where $\lambda>0$ is a positive parameter, $k:|\times| \rightarrow \mathbb{R}$ is a kernel function such that $k \in W^{m, 1}(|\times|), m$ is a positive integer with $m \geq 1$, and $f: l \times \mathbb{R}^{m+1} \rightarrow[0,+\infty$ [ is an $\mathrm{L}^{1}$-Carathéodory function.

The existence of solutions for these Hammerstein equations is obtained by fixed point index theory on new type of cones. Therefore some assumptions must hold only for, at least, one of the derivatives of the kernel or, even, for the kernel on a subset of the domain. Assuming some asymptotic conditions on the nonlinearity $f$, we get sufficient conditions for multiplicity of solutions.

Two examples will illustrate the potentialities of the main results, namely the fact that the kernel function and/or some derivatives may only be positive on some subintervals, which can degenerate to a point. Moreover, an application of our method to general Lidstone problems improves the existent results in the literature in this field.

MSC: 34B08; 34B10; 34B15; 34B18; 34B27

Keywords: Hammerstein equations; Nonlinear boundary value problems; Parameter dependence; Degree theory; Fixed points in cones

\section{Introduction}

In this work we will study the existence and multiplicity of fixed points of the integral operator

$$
\mathcal{T} u(t)=\lambda \int_{0}^{T} k(t, s) f\left(s, u(s), u^{\prime}(s), \ldots, u^{(m)}(s)\right) \mathrm{d} s, \quad t \in[0, T] \equiv I,
$$

where

- $\lambda>0$ is a positive parameter,

- $k: I \times I \rightarrow \mathbb{R}$ is a kernel function such that $k \in W^{m, 1}(I \times I)$,

(c) The Author(s) 2019. This article is distributed under the terms of the Creative Commons Attribution 4.0 International License (http://creativecommons.org/licenses/by/4.0/), which permits unrestricted use, distribution, and reproduction in any medium, provided you give appropriate credit to the original author(s) and the source, provide a link to the Creative Commons license, and indicate if changes were made. 
- $m$ is a positive integer with $m \geq 1$, and

- $f: I \times \mathbb{R}^{m+1} \rightarrow\left[0,+\infty\left[\right.\right.$ is an $\mathrm{L}^{1}$-Carathéodory function.

The solvability of this type of integral equations, known as Hammerstein equations (see [12]), has been considered by many authors. In fact they have become both a generalization of differential equations and boundary value problems and a main field for applications of methods and techniques of nonlinear analysis, as it can be seen, for instance, in $[1-3,7$, $11,13-17,21]$.

In [4], the authors consider a third order three-point boundary value problem, whose solutions are the fixed points of the integral operator

$$
T u(t)=\lambda \int_{0}^{1} G(t, s) f\left(s, u(s), u^{\prime}(s), u^{\prime \prime}(s)\right) \mathrm{d} s, \quad t \in[0,1],
$$

where $G(t, s)$ is an explicit Green's function, verifying some adequate properties such that $G(t, s)$ and $\frac{\partial G}{\partial t}(t, s)$ are bounded and nonnegative in the square $[0,1] \times[0,1]$, but $\frac{\partial^{2} G}{\partial t^{2}}(t, s)$ could change sign, being nonnegative in a subset of the square.

In [8], a generalized Hammerstein equation is studied

$$
u(t)=\int_{0}^{1} k(t, s) g(s) f\left(s, u(s), u^{\prime}(s), \ldots, u^{(m)}(s)\right) \mathrm{d} s
$$

with $k:[0,1]^{2} \rightarrow \mathbb{R}$ a kernel function such that $k \in W^{m, 1}\left([0,1]^{2}\right), m \geq 1$ is integer, $g:[0,1] \rightarrow[0, \infty)$ is nonnegative almost everywhere in $t \in[0,1]$ nonnegative, and $f:$ $[0,1] \times \mathbb{R}^{m+1} \rightarrow[0, \infty)$ is an $\mathrm{L}^{\infty}$-Carathéodory function. Moreover, both the kernel $k(t, s)$ and its derivatives $\frac{\partial^{i} k}{\partial t^{i}}(t, s)$, for $i=1, \ldots, m$, are bounded and nonnegative on the square $[0,1] \times[0,1]$.

Our work generalizes the existing results in the literature introducing a new type of cone:

$$
\begin{aligned}
K= & \left\{u \in \mathcal{C}^{m}(I, \mathbb{R}): u^{(i)}(t) \geq 0, t \in\left[m_{i}, n_{i}\right], i \in J ;\right. \\
& \left.\min _{t \in\left[a_{j}, b_{j}\right]} u^{(j)}(t) \geq \xi_{j}\left\|u^{(j)}\right\|_{\left[c_{j}, d_{j}\right]}, j \in J_{1}\right\},
\end{aligned}
$$

where

$$
\left\|u^{(j)}\right\|_{\left[c_{j}, d_{j}\right]}:=\max _{t \in\left[c_{j}, d_{j}\right]}\left|u^{(j)}(t)\right|
$$

$J \equiv\{0,1, \ldots, m\}$ and $J_{1} \subset J_{0} \subset J, J_{1} \neq \varnothing$.

We note that the non-negativeness of the functions and their derivatives may happen only on a subinterval, possibly degenerate (that is reduced to a point), and, as $J_{1} \subset J, J_{1} \neq \varnothing$, the second property can hold, locally, only for a restricted number of derivatives, including the function itself. This way, it is not required, as it was usual, that $k(t, s)$ and $\frac{\partial^{i} k}{\partial t^{i}}(t, s)$ have constant sign on the square of definition.

Another important novelty is that, in the second property of the cone, we are considering the norm of the functions on a subset of the domain and not on the whole interval $[0, T]$. Moreover, as we will see, the two subintervals involved in this condition, $\left[a_{j}, b_{j}\right]$ and $\left[c_{j}, d_{j}\right]$, must have nonempty intersection but are not required to satisfy any other inclusion property (that is, it may occur that $\left[a_{j}, b_{j}\right] \not \subset\left[c_{j}, d_{j}\right]$ and $\left[c_{j}, d_{j}\right] \not \subset\left[a_{j}, b_{j}\right]$ ). 
In particular, the second property in the cone (which ensures that the minimum of the function and the derivatives on some interval is bigger than its norm on another interval) will be given by certain inequalities (introduced in $\left(H_{4}\right)$ ) that both the kernel and its derivatives must hold. However, contrary to recent references such as [8], the bounds must hold only for, at least, one of the derivatives of the kernel or, even, for the kernel on a subset of the domain.

This paper is organized in the following way: Sect. 2 contains the main assumptions, the definition of the new cone and some properties on the integral operator. In Sect. 3, the existence results are obtained with several asymptotic assumptions on $f$ of the sublinear or superlinear type, near 0 or $+\infty$. Section 4 presents existence and multiplicity results applying fixed point index theory. Section 5 has two examples to illustrate our main results and, moreover, to emphasize the importance that $\left(H_{4}\right)$ holds only for some derivatives and that the subsets could be reduced to a point. Last section contains an application to $2 n$-th order Lidstone problems, giving new sufficient conditions for the solvability of this kind of problems. In fact, our method allows that the nonlinearities may depend on derivatives of even and odd order, which is new in the literature on this type of problems, as it can be seen, for instance, in $[5,18,23,26]$. In this way, our results fill some gaps and improve the study of Lidstone and complementary Lidstone problems.

\section{Hypothesis and auxiliary results}

Let us consider $E=\mathcal{C}^{m}(I, \mathbb{R})$ and the norm $\|u\|=\max \left\{\left\|u^{(i)}\right\|_{\infty}, i \in J\right\}$, with $\|v\|_{\infty}=\sup _{t \in I}|v(t)|$. It is clear that $(E,\|\cdot\|)$ is a Banach space.

Throughout this paper we will make the following assumptions:

$\left(H_{1}\right)$ The kernel function $k: I \times I \rightarrow \mathbb{R}$ is such that $k \in W^{m, 1}(I \times I)$, with $m \geq 1$. Moreover, for $i=0, \ldots, m-1$, it holds that, for every $\varepsilon>0$ and every fixed $\tau \in I$, there exists some $\delta>0$ such that $|t-\tau|<\delta$ implies that

$$
\left|\frac{\partial^{i} k}{\partial t^{i}}(t, s)-\frac{\partial^{i} k}{\partial t^{i}}(\tau, s)\right|<\varepsilon \quad \text { for a.e. } s \in I .
$$

Finally, for the $m$ th derivative of the kernel, it holds that, for every $\varepsilon>0$ and every fixed $\tau \in I$, there exist a set $Z_{\tau} \in I$ with measure equal to zero and some $\delta>0$ such that $|t-\tau|<\delta$ implies that

$$
\left|\frac{\partial^{m} k}{\partial t^{m}}(t, s)-\frac{\partial^{m} k}{\partial t^{m}}(\tau, s)\right|<\varepsilon, \quad \forall s \in I \backslash Z_{\tau} \text { such that } s<\min \{t, \tau\} \text { or } s>\max \{t, \tau\} .
$$

$\left(H_{2}\right)$ For each $i \in J_{0} \subset J, J_{0} \neq \varnothing$, there exists a subinterval $\left[m_{i}, n_{i}\right]$ such that

$$
\frac{\partial^{i} k}{\partial t^{i}}(t, s) \geq 0 \quad \text { for all } t \in\left[m_{i}, n_{i}\right], s \in I .
$$

It is possible that the interval is degenerated, that is, $m_{i}=n_{i}$.

$\left(H_{3}\right)$ For all $i \in J$, there exist positive functions $h_{i} \in \mathrm{L}^{1}(I)$ such that

$$
\left|\frac{\partial^{i} k}{\partial t^{i}}(t, s)\right| \leq h_{i}(s) \quad \text { for all } t \in I \text { and a.e. } s \in I \text {. }
$$


$\left(H_{4}\right)$ For each $j \in J_{1} \subset J_{0}, J_{1} \neq \varnothing$, there exist subintervals $\left[a_{j}, b_{j}\right] \subset\left[m_{j}, n_{j}\right]$ and $\left[c_{j}, d_{j}\right]$, with $\left[a_{j}, b_{j}\right] \cap\left[c_{j}, d_{j}\right] \neq \varnothing$, positive functions $\phi_{j}: I \rightarrow[0, \infty)$, and constants $\xi_{j} \in(0,1)$ such that

$$
\left|\frac{\partial^{j} k}{\partial t^{j}}(t, s)\right| \leq \phi_{j}(s) \quad \text { for all } t \in\left[c_{j}, d_{j}\right] \text { and a.e. } s \in I
$$

and

$$
\frac{\partial^{j} k}{\partial t^{j}}(t, s) \geq \xi_{j} \phi_{j}(s) \quad \text { for all } t \in\left[a_{j}, b_{j}\right] \text { and a.e. } s \in I \text {. }
$$

Moreover, $\phi_{j} \in \mathrm{L}^{1}(I)$ satisfies that $\int_{a_{j}}^{b_{j}} \phi_{j}(s) \mathrm{d} s>0$.

$\left(H_{5}\right)$ There exists $i_{0} \in J_{0}$ such that either $\left[c_{i_{0}}, d_{i_{0}}\right] \equiv I$ or $\left[m_{i_{0}}, n_{i_{0}}\right] \equiv I$ and, moreover, $\left\{0,1, \ldots, i_{0}\right\} \subset J_{0}$.

$\left(H_{6}\right)$ The nonlinearity $f: I \times \mathbb{R}^{m+1} \rightarrow[0, \infty)$ satisfies $\mathrm{L}^{1}$-Carathéodory conditions, that is,

- $f\left(\cdot, x_{0}, \ldots, x_{m}\right)$ is measurable for each $\left(x_{0}, \ldots, x_{m}\right)$ fixed.

- $f(t, \cdot, \ldots, \cdot)$ is continuous for a.e. $t \in I$.

- For each $r>0$, there exists $\varphi_{r} \in \mathrm{L}^{1}(I)$ such that

$$
f\left(t, x_{0}, \ldots, x_{m}\right) \leq \varphi_{r}(t), \quad \forall\left(x_{0}, \ldots, x_{m}\right) \in(-r, r)^{m+1} \text {, a.e. } t \in I .
$$

$\left(H_{7}\right)$ Functions $h_{i}$ defined in $\left(H_{3}\right)$ and $\varphi_{r}$ defined in $\left(H_{6}\right)$ are such that $h_{i} \varphi_{r} \in \mathrm{L}^{1}(I)$ for every $i \in J$ and $r>0$.

We will look for fixed points of operator $\mathcal{T}$ on a suitable cone on the Banach space $E$. We recall that a cone $K$ is a closed and convex subset of $E$ satisfying the two following properties:

- If $x \in K$, then $\lambda x \in K$ for all $\lambda \geq 0$.

- $K \cap(-K)=\{0\}$.

In particular, taking into account the properties satisfied by the kernel $k$, we define

$$
\begin{aligned}
K= & \left\{u \in \mathcal{C}^{m}(I, \mathbb{R}): u^{(i)}(t) \geq 0, t \in\left[m_{i}, n_{i}\right], i \in J_{0} ;\right. \\
& \left.\min _{t \in\left[a_{j}, b_{j}\right]} u^{(j)}(t) \geq \xi_{j}\left\|u^{(j)}\right\|_{\left[c_{j}, d_{j}\right]}, j \in J_{1}\right\} .
\end{aligned}
$$

Lemma 1 Hypothesis $\left(H_{5}\right)$ warrants that $K$ is a cone in $E$.

Proof We need to verify that $K$ is a closed and convex subset of $\mathcal{C}^{m}(I, \mathbb{R})$ and that it satisfies the two properties which characterize cones in a Banach space.

First of all, from the definition of $K$, it is clear that it is closed. We will see that it is convex. For $u, v \in K$ and $\lambda \in(0,1)$, it is clear that

$$
(1-\lambda) u^{(i)}(t)+\lambda v^{(i)}(t) \geq 0 \quad \text { for } t \in\left[m_{i}, n_{i}\right], i \in J_{0} .
$$


In addition, for $j \in J_{1}$,

$$
\begin{aligned}
\min _{t \in\left[a_{j}, b_{j}\right]}\left((1-\lambda) u^{(j)}(t)+\lambda v^{(j)}(t)\right) & \geq(1-\lambda) \min _{t \in\left[a_{j}, b_{j}\right]} u^{(j)}(t)+\lambda \min _{t \in\left[a_{j}, b_{j}\right]} v^{(j)}(t) \\
& \geq(1-\lambda) \xi_{j}\left\|u^{(j)}\right\|_{\left[c_{j}, d_{j}\right]}+\lambda \xi_{j}\left\|v^{(j)}\right\|_{\left[c_{j}, d_{j}\right]} \\
& =\xi_{j}\left(\left\|(1-\lambda) u^{(j)}\right\|_{\left[c_{j}, d_{j}\right]}+\left\|\lambda v^{(j)}\right\|_{\left[c_{j}, d_{j}\right]}\right) \\
& \geq \xi_{j}\left\|(1-\lambda) u^{(j)}+\lambda v^{(j)}\right\|_{\left[c_{j}, d_{j}\right]} .
\end{aligned}
$$

Thus, $(1-\lambda) u+\lambda v \in K$.

Moreover, from the definition of $K$, it is trivial to check that if $x \in K$, then $\lambda x \in K$ for all $\lambda \geq 0$.

Now, to prove that $K \cap(-K)=\{0\}$, we will distinguish between two different cases:

(I) There exists $i_{0} \in J_{0}$ such that $\left[m_{i_{0}}, n_{i_{0}}\right] \equiv I$.

Suppose that $u,-u \in K$. Then $u^{\left(i_{0}\right)}(t) \geq 0$ and $-u^{\left(i_{0}\right)}(t) \geq 0$ for all $t \in I$, which implies that $u^{\left(i_{0}\right)} \equiv 0$ on $I$. If $i_{0} \geq 1, u^{\left(i_{0}-1\right)}$ is constant on $I$.

Now, we have that $u^{\left(i_{0}-1\right)}(t) \geq 0$ and $-u^{\left(i_{0}-1\right)}(t) \geq 0$ for all $t \in\left[c_{i_{0}-1}, d_{i_{0}-1}\right]$, that is, $u^{\left(i_{0}-1\right)} \equiv 0$ on $\left[c_{i_{0}-1}, d_{i_{0}-1}\right]$. Then, since $u^{\left(i_{0}-1\right)}$ is constant on $I$, we deduce that $u^{\left(i_{0}-1\right)} \equiv 0$ on $I$.

Using the same argument repeatedly, we conclude that $u \equiv 0$ on $I$. In this way, we have proved that $K \cap(-K)=\{0\}$.

(II) There exists $i_{0} \in J_{0}$ such that $\left[c_{i_{0}}, d_{i_{0}}\right] \equiv I$.

Suppose again that $u,-u \in K$. Then, from the fact that

$$
\min _{t \in\left[a_{i_{0}}, b_{i_{0}}\right]} u^{\left(i_{0}\right)}(t) \geq \xi_{i_{0}}\left\|u^{\left(i_{0}\right)}\right\|_{I} \quad \text { and } \min _{t \in\left[a_{i_{0}}, b_{i_{0}}\right]}\left(-u^{\left(i_{0}\right)}(t)\right) \geq \xi_{i_{0}}\left\|u^{\left(i_{0}\right)}\right\|_{I}
$$

it is deduced that $\left\|u^{\left(i_{0}\right)}\right\|_{I}=0$, which implies that $u^{\left(i_{0}\right)} \equiv 0$ on $I$. Now, following the same arguments as in Case (I), we deduce the result.

In the next section, we will ensure the existence of fixed points of operator $\mathcal{T}$. Before that, we need to prove that operator $\mathcal{T}$ is compact.

Lemma 2 If $\left(H_{1}\right)-\left(H_{7}\right)$ hold, then operator $\mathcal{T}$ maps the cone into itself and is completely continuous.

Proof Using standard techniques, it is easy to prove that $\mathcal{T}$ is well defined, $\mathcal{T}(K) \subset K$, and $\mathcal{T}$ is continuous in $\mathcal{C}^{m}(I, \mathbb{R})$.

We will see now that $\mathcal{T}$ is completely continuous.

Consider

$$
B=\{u \in E ;\|u\| \leq r\}
$$

It is obvious that $T(B)$ is uniformly bounded in $\mathcal{C}^{m}(I)$. 
Let us prove that $\mathcal{T}(B)$ is equicontinuous in $\mathcal{C}^{m}(I)$. Let $t_{2} \in I$ be fixed. Then, for every $\varepsilon>0$, take $\delta>0$ given in $\left(H_{1}\right)$, and for $i=0, \ldots, m-1$, it holds that $\left|t_{1}-t_{2}\right|<\delta$ implies that

$$
\begin{aligned}
\left|(\mathcal{T} u)^{(i)}\left(t_{1}\right)-(\mathcal{T} u)^{(i)}\left(t_{2}\right)\right| & \leq \lambda \int_{0}^{T}\left|\frac{\partial^{i} k}{\partial t^{i}}\left(t_{1}, s\right)-\frac{\partial^{i} k}{\partial t^{i}}\left(t_{2}, s\right)\right| f\left(s, u(s), \ldots, u^{(m)}(s)\right) \mathrm{d} s \\
& \leq \lambda \int_{0}^{T}\left|\frac{\partial^{i} k}{\partial t^{i}}\left(t_{1}, s\right)-\frac{\partial^{i} k}{\partial t^{i}}\left(t_{2}, s\right)\right| \varphi_{r}(s) \mathrm{d} s \leq \varepsilon \lambda \int_{0}^{T} \varphi_{r}(s) \mathrm{d} s,
\end{aligned}
$$

and, since $\varphi_{r} \in \mathrm{L}^{1}(I)$, it is clear that there exists a positive constant $\kappa_{1}$ such that

$$
\left|(\mathcal{T} u)^{(i)}\left(t_{1}\right)-(\mathcal{T} u)^{(i)}\left(t_{2}\right)\right|<\kappa_{1} \varepsilon
$$

for all $u \in B$.

On the other hand, for the $m$ th derivative, for every $\varepsilon>0$, take $\delta>0$ given in $\left(H_{1}\right)$ and $\left|t_{1}-t_{2}\right|<\delta, t_{1}<t_{2}$, implies that

$$
\begin{aligned}
\left|(\mathcal{T} u)^{(m)}\left(t_{1}\right)-(\mathcal{T} u)^{(m)}\left(t_{2}\right)\right| \leq & \lambda \int_{0}^{T}\left|\frac{\partial^{m} k}{\partial t^{m}}\left(t_{1}, s\right)-\frac{\partial^{m} k}{\partial t^{m}}\left(t_{2}, s\right)\right| f\left(s, u(s), \ldots, u^{(m)}(s)\right) \mathrm{d} s \\
\leq & \lambda \int_{0}^{T}\left|\frac{\partial^{m} k}{\partial t^{m}}\left(t_{1}, s\right)-\frac{\partial^{m} k}{\partial t^{m}}\left(t_{2}, s\right)\right| \varphi_{r}(s) \mathrm{d} s \\
= & \lambda \int_{0}^{t_{1}}\left|\frac{\partial^{m} k}{\partial t^{m}}\left(t_{1}, s\right)-\frac{\partial^{m} k}{\partial t^{m}}\left(t_{2}, s\right)\right| \varphi_{r}(s) \mathrm{d} s \\
& +\lambda \int_{t_{1}}^{t_{2}}\left|\frac{\partial^{m} k}{\partial t^{m}}\left(t_{1}, s\right)-\frac{\partial^{m} k}{\partial t^{m}}\left(t_{2}, s\right)\right| \varphi_{r}(s) \mathrm{d} s \\
& +\lambda \int_{t_{2}}^{T}\left|\frac{\partial^{m} k}{\partial t^{m}}\left(t_{1}, s\right)-\frac{\partial^{m} k}{\partial t^{m}}\left(t_{2}, s\right)\right| \varphi_{r}(s) \mathrm{d} s .
\end{aligned}
$$

From $\left(H_{1}\right)$, it is clear that first and third integrals in the last term of previous expression can be arbitrarily small when $\left|t_{1}-t_{2}\right|<\delta$. Moreover, $\left|\frac{\partial^{m} k}{\partial t^{m}}\left(t_{1}, \cdot\right)-\frac{\partial^{m} k}{\partial t^{m}}\left(t_{2}, \cdot\right)\right| \varphi_{r}(\cdot) \in \mathrm{L}^{1}\left[t_{1}, t_{2}\right]$, and so there exists some $\delta^{\prime}>0$ such that

$$
\lambda \int_{t_{1}}^{t_{2}}\left|\frac{\partial^{m} k}{\partial t^{m}}\left(t_{1}, s\right)-\frac{\partial^{m} k}{\partial t^{m}}\left(t_{2}, s\right)\right| \varphi_{r}(s) \mathrm{d} s<\varepsilon
$$

when $\left|t_{1}-t_{2}\right|<\delta^{\prime}$.

Therefore it is clear that, for $\left|t_{1}-t_{2}\right|<\min \left\{\delta, \delta^{\prime}\right\}, t_{1}<t_{2}$, there exists a positive constant $\kappa_{2}$ such that

$$
\left|(\mathcal{T} u)^{(m)}\left(t_{1}\right)-(\mathcal{T} u)^{(m)}\left(t_{2}\right)\right|<\kappa_{2} \varepsilon
$$

for all $u \in B$.

Analogously, when $\left|t_{1}-t_{2}\right|<\delta, t_{1}>t_{2}$, there exists some some positive constant $\kappa_{3}$ such that

$$
\left|(\mathcal{T} u)^{(m)}\left(t_{1}\right)-(\mathcal{T} u)^{(m)}\left(t_{2}\right)\right|<\kappa_{3} \varepsilon
$$

for all $u \in B$. 
We have proved the pointwise equicontinuity on $I$. Moreover, since $I$ is compact, pointwise equicontinuity is equivalent to uniform equicontinuity.

This way, we can affirm that $\mathcal{T}(B)$ is equicontinuous in $\mathcal{C}^{m}(I)$.

From Ascoli-Arzelà theorem, we deduce that $\mathcal{T}(B)$ is relatively compact in $\mathcal{C}^{m}(I)$. Therefore, $\mathcal{T}$ is completely continuous.

\section{Existence results}

In this section, we present two different methods to prove the existence of fixed points of operator (1). As we will see with the examples given in the next section, our two methods are not comparable, but complementary, in the sense that some examples can be solved using one of the methods (but not the other one) and vice-versa.

The first method is based on the fixed point index theory on arbitrary open sets which might be, in particular, unbounded (see [9] for details). To develop this method, we need to introduce some notation

$$
\begin{aligned}
& \Lambda^{i}:=\int_{0}^{T} h_{i}(s) \mathrm{d} s, \quad \Lambda_{i}:=\int_{a_{i}}^{b_{i}} \xi_{i} \phi_{i}(s) \mathrm{d} s, \\
& \bar{\Lambda}:=(m+1) \max \left\{\Lambda^{i}: i \in J\right\} \quad \text { and } \quad \underline{\Lambda}:=\max \left\{\xi_{i} \Lambda_{i}: i \in J_{1}\right\} .
\end{aligned}
$$

Moreover, we will compute the following limits at 0 and $\infty$ :

$$
f_{0}:=\liminf _{\left|x_{0}\right|, \ldots,\left|x_{m}\right| \rightarrow 0} \min _{t \in I} \frac{f\left(t, x_{0}, \ldots, x_{m}\right)}{\left|x_{0}\right|+\cdots+\left|x_{m}\right|}, \quad f^{\infty}:=\limsup _{\left|x_{0}\right|, \ldots,\left|x_{m}\right| \rightarrow \infty} \max _{t \in I} \frac{f\left(t, x_{0}, \ldots, x_{m}\right)}{\left|x_{0}\right|+\cdots+\left|x_{m}\right|} .
$$

Our main result is the following one.

Theorem 3 Let $\left(H_{1}\right)-\left(H_{7}\right)$ hold and assume that $\bar{\Lambda} f^{\infty}<\underline{\Lambda} f_{0}$. Then, for all $\lambda \in\left(\frac{1}{\underline{\Lambda} f_{0}}, \frac{1}{\bar{\Lambda} f^{\infty}}\right)$, operator $\mathcal{T}$ has a fixed point in the cone $K$, which is not a trivial solution.

Proof Fix $\lambda \in\left(\frac{1}{\underline{\Lambda} f_{0}}, \frac{1}{\bar{\Lambda} f^{\infty}}\right)$ and let $0<\varepsilon<f_{0}$ be such that $\frac{1}{\underline{\Lambda}\left(f_{0}-\varepsilon\right)} \leq \lambda \leq \frac{1}{\bar{\Lambda}\left(f^{\infty}+\varepsilon\right)}$.

Then there exists some $\delta>0$ such that, when $\|u\| \leq \delta$, the following inequality holds:

$$
f\left(t, u(t), \ldots, u^{(m)}(t)\right)>\left(f_{0}-\varepsilon\right)\left(|u(t)|+\cdots+\left|u^{(m)}(t)\right|\right), \quad \forall t \in I .
$$

Define now $\Omega_{\delta}=\{u \in K ;\|u\|<\delta\}$ and let $u \in \partial \Omega_{\delta}$. We will prove that $\mathcal{T} u \npreceq u$. Indeed, for $t \in\left[a_{i}, b_{i}\right]$ and $j \in J_{1}$, the following inequalities are satisfied:

$$
\begin{aligned}
(\mathcal{T} u)^{(j)}(t) & =\lambda \int_{0}^{T} \frac{\partial^{j} k}{\partial t^{j}}(t, s) f\left(s, u(s), \ldots, u^{(m)}(s)\right) \mathrm{d} s \\
& \geq \lambda \int_{a_{j}}^{b_{j}} \frac{\partial^{j} k}{\partial t^{j}}(t, s) f\left(s, u(s), \ldots, u^{(m)}(s)\right) \mathrm{d} s \\
& \geq \lambda \int_{a_{j}}^{b_{j}} \xi_{j} \phi_{j}(s) f\left(s, u(s), \ldots, u^{(m)}(s)\right) \mathrm{d} s \\
& >\lambda \int_{a_{j}}^{b_{j}} \xi_{j} \phi_{j}(s)\left(f_{0}-\varepsilon\right)\left(|u(s)|+\cdots+\left|u^{(m)}(s)\right|\right) \mathrm{d} s
\end{aligned}
$$




$$
\begin{aligned}
& \geq \lambda\left(f_{0}-\varepsilon\right) \xi_{j}\left\|u^{(j)}\right\|_{\left[a_{j}, b_{j}\right]} \int_{a_{j}}^{b_{j}} \xi_{j} \phi_{j}(s) \mathrm{d} s \\
& =\lambda\left(f_{0}-\varepsilon\right) \xi_{j}\left\|u^{(j)}\right\|_{\left[a_{j}, b_{j}\right]} \Lambda_{j} \geq \lambda\left(f_{0}-\varepsilon\right) \Lambda_{j} \xi_{j} u^{(j)}(t) .
\end{aligned}
$$

Taking into account the definition of $\underline{\Lambda}$, we deduce that, for some $j \in J_{1},(\mathcal{T} u)^{(j)}(t)>u^{(j)}(t)$ for all $t \in\left[a_{j}, b_{j}\right]$. Therefore $\mathcal{T} u \npreceq u$ and, from [10, Theorem 2.3.3], we deduce that $i_{K}\left(\mathcal{T}, \Omega_{\delta}\right)=0$.

By the same token, there is some value $\tilde{C}>0$ such that if $\min \left\{\left|u^{(i)}(t)\right|: i \in J\right\} \geq \tilde{C}$, then

$$
f\left(t, u(t), \ldots, u^{(m)}(t)\right) \leq\left(f^{\infty}+\varepsilon\right)\left(|u(t)|+\cdots+\left|u^{(m)}(t)\right|\right) \leq(m+1)\left(f^{\infty}+\varepsilon\right)\|u\|, \quad \forall t \in I .
$$

Take now $C>\{\delta, \tilde{C}\}$ and let $\Omega_{C}=\bigcup_{i=0}^{m}\left\{u \in K: \min _{t \in I}\left|u^{(i)}(t)\right|<C\right\}$. Since $\Omega_{C}$ is an unbounded subset of the cone $K$, we need to check that the fixed point index of $\mathcal{T}$ with respect to $\Omega_{C}, i_{K}\left(\mathcal{T}, \Omega_{C}\right)$, can be defined. In particular, $i_{K}\left(\mathcal{T}, \Omega_{C}\right)$ is well defined only if the set of fixed points of $\mathcal{T}$ in $\Omega_{C}$, that is, $(I-\mathcal{T})^{-1}(\{0\}) \cap \Omega_{C}$, is compact (see [9]).

Clearly, the continuity of $(I-\mathcal{T})$ implies that $(I-\mathcal{T})^{-1}(\{0\}) \cap \Omega_{C}$ is closed.

On the other hand, we observe that if $(I-\mathcal{T})^{-1}(\{0\}) \cap \Omega_{C}$ is unbounded, then we would have an infinite number of fixed points of $\mathcal{T}$ in $\Omega_{C}$, which would be, in particular, fixed points of $\mathcal{T}$ in the cone $K$. Thus, it can be supposed the existence of some positive constant $M$ such that $\|u\|<M$ for all $u \in(I-\mathcal{T})^{-1}(\{0\}) \cap \Omega_{C}$.

Finally, the proof that $(I-\mathcal{T})^{-1}(\{0\}) \cap \Omega_{C}$ is equicontinuous is analogous to the one made for Lemma 2.

Now, we will prove that $\|\mathcal{T} u\| \leq\|u\|$ for all $u \in \partial \Omega_{C}$. Take $u \in \partial \Omega_{C}$, in other words, $u \in K$ satisfying that $\min \left\{\min _{t \in I}\left|u^{(i)}(t)\right|: i \in J\right\}=C$. Then, for $i \in J$,

$$
\begin{aligned}
\left|(\mathcal{T} u)^{(i)}(t)\right| & \leq \lambda \int_{0}^{T}\left|\frac{\partial^{i} k}{\partial t^{i}}(t, s)\right| f\left(s, u(s), \ldots, u^{(m)}(s)\right) \mathrm{d} s \\
& \leq \lambda \int_{0}^{T} h_{i}(s) f\left(s, u(s), \ldots, u^{(m)}(s)\right) \mathrm{d} s \\
& \leq(m+1) \lambda \int_{0}^{T} h_{i}(s)\left(f^{\infty}+\varepsilon\right)\|u\| \mathrm{d} s \\
& =(m+1) \lambda\left(f^{\infty}+\varepsilon\right)\|u\| \Lambda^{i} \leq \lambda\left(f^{\infty}+\varepsilon\right)\|u\| \bar{\Lambda} \leq\|u\| .
\end{aligned}
$$

As a consequence, $\|\mathcal{T} u\| \leq\|u\|$ and so, from [10, Theorem 2.3.3], we deduce that $i_{K}\left(\mathcal{T}, \Omega_{C}\right)=1$.

Therefore, we can ensure the existence of a fixed point of operator $\mathcal{T}$ in $\bar{\Omega}_{C} \backslash \Omega_{\delta}$.

We present now another approach to prove both existence and multiplicity of fixed points of the integral operator $\mathcal{T}$. This method, which is not comparable with the previous one, is based on the fixed point index theory on bounded sets.

First of all, we need to introduce the following sets:

$$
\begin{aligned}
& K_{\rho}=\{u \in K ;\|u\|<\rho\}, \\
& V_{\rho}=\left\{u \in K: \min _{t \in\left[a_{i}, b_{i}\right]} u^{(i)}(t)<\rho, i \in J_{2},\left\|u^{(i)}\right\|_{\infty}<\rho, i \in J \backslash J_{2}\right\},
\end{aligned}
$$

where $J_{2}=\left\{i \in J:\left[c_{i}, d_{i}\right] \equiv I\right\}$. 
Moreover, to ensure that the sets $K_{\rho}$ and $V_{\rho}$ are not the same, we need to change condition $\left(H_{5}\right)$ into

$\left(\widetilde{H}_{5}\right)$ There exists $i_{0} \in\{0, \ldots, m\}$ such that $\left[c_{i_{0}}, d_{i_{0}}\right] \equiv I$ and, moreover, $\left\{0,1, \ldots, i_{0}\right\} \subset J_{0}$. In this situation, it occurs that $J_{2} \neq \emptyset$ and therefore $K_{\rho} \subsetneq V_{\rho} \subsetneq K_{\frac{\rho}{c}}$, being

$$
c=\min \left\{\xi_{i}: i \in J_{2}\right\}
$$

We formulate sufficient conditions under which the index of the previous sets is either 1 or 0 .

Lemma 4 Let $\frac{1}{N}=\max \left\{\sup _{t \in I} \int_{0}^{T}\left|\frac{\partial^{i} k}{\partial t^{i}}(t, s)\right| \mathrm{d} s: i \in J\right\}$ and

$$
f^{\rho}=\sup \left\{\frac{f\left(t, x_{0}, \ldots, x_{m}\right)}{\rho} ; t \in I, x_{i} \in[-\rho, \rho], i \in J\right\} \text {. }
$$

If there is some value $\rho>0$ for which

$$
\lambda \frac{f^{\rho}}{N}<1
$$

then $i_{K}\left(\mathcal{T}, K_{\rho}\right)=1$.

Proof Let us see that given $u \in \partial K_{\rho}$ and $\mu \geq 1$, it occurs that $\mathcal{T} u \neq \mu u$.

Suppose that previous assertion is not true. Then there exist $u \in \partial K_{\rho}$ and $\mu \geq 1$ for which

$$
\mu u^{(i)}(t)=\lambda \int_{0}^{T} \frac{\partial^{i} k}{\partial t^{i}}(t, s) f\left(s, u(s), \ldots, u^{(m)}(s)\right) \mathrm{d} s .
$$

Now, if we compute the supremum for $t \in I$, we get the following inequalities:

$$
\begin{aligned}
\mu\left\|u^{(i)}\right\|_{\infty} & \leq \lambda \sup _{t \in I} \int_{0}^{T}\left|\frac{\partial^{i} k}{\partial t^{i}}(t, s)\right| f\left(s, u(s), \ldots, u^{(m)}(s)\right) \mathrm{d} s \\
& \leq \lambda \rho f^{\rho} \sup _{t \in I} \int_{0}^{T}\left|\frac{\partial^{i} k}{\partial t^{i}}(t, s)\right| \mathrm{d} s \leq \lambda \rho \frac{f^{\rho}}{N}<\rho .
\end{aligned}
$$

Consequently, $\mu \rho=\mu \max \left\{\left\|u^{(i)}\right\|_{\infty}: i \in J\right\}<\rho$, which contradicts that $\mu \geq 1$. Thus (see $\left[9\right.$, Corollary 7.4]) $i_{K}\left(\mathcal{T}, K_{\rho}\right)=1$.

Lemma 5 For $i \in J_{1}$, let $\frac{1}{M_{i}}=\inf _{t \in\left[a_{i}, b_{i}\right]} \int_{a_{i}}^{b_{i}} \frac{\partial^{i} k}{\partial t^{i}}(t, s) \mathrm{d} s$, and

$$
f_{\rho}^{i}=\inf \left\{\frac{f\left(t, x_{0}, \ldots, x_{m}\right)}{\rho}: t \in\left[a_{i}, b_{i}\right], x_{j} \in\left[0, \frac{\rho}{\xi_{j}}\right], j \in J_{2}, x_{k} \in[0, \rho], k \in J \backslash J_{2}\right\} .
$$

If there exist some constant $\rho>0$ and some index $i_{0} \in J_{1}$ such that

$$
\lambda \frac{f_{\rho}^{i_{0}}}{M_{i_{0}}}>1
$$

then $i_{K}\left(\mathcal{T}, V_{\rho}\right)=0$. 
Proof Take $e(t)=1$ (clearly $e \in K \backslash\{0\})$. We will se that $u \neq \mathcal{T} u+\alpha e$ for all $u \in \partial V_{\rho}$ and $\alpha>0$.

Assume, on the contrary, that there exist $u \in \partial V_{\rho}$ and $\alpha>0$ such that $u=\mathcal{T} u+\alpha$. Then, for $t \in\left[a_{i_{0}}, b_{i_{0}}\right]$,

$$
\begin{aligned}
u^{\left(i_{0}\right)}(t) & \geq \lambda \int_{0}^{T} \frac{\partial^{i_{0}} k}{\partial t^{i_{0}}}(t, s) f\left(s, u(s), \ldots, u^{(m)}(s)\right) \mathrm{d} s \\
& \geq \lambda \int_{a_{i_{0}}}^{b_{i_{0}}} \frac{\partial^{i_{0}} k}{\partial t^{i_{0}}}(t, s) f\left(s, u(s), \ldots, u^{(m)}(s)\right) \mathrm{d} s \\
& \geq \lambda \rho f_{\rho}^{i_{0}} \int_{a_{i_{0}}}^{b_{i_{0}}} \frac{\partial^{i_{0}} k}{\partial t^{i_{0}}}(t, s) \mathrm{d} s>\rho .
\end{aligned}
$$

Therefore, $u^{\left(i_{0}\right)}(t)>\rho$ for $t \in\left[a_{i_{0}}, b_{i_{0}}\right]$, which is a contradiction. As a consequence (see $\left[9\right.$, Corollary 7.11]), $i_{K}\left(\mathcal{T}, V_{\rho}\right)=0$.

Combining the previous lemmas, it is possible to obtain some conditions under which operator $\mathcal{T}$ has one or even multiple fixed points.

Theorem 6 Assume that conditions $\left(H_{1}\right)-\left(H_{4}\right),\left(\widetilde{H}_{5}\right)$, and $\left(H_{6}\right)-\left(H_{7}\right)$ hold and let $c$ be defined in (3). If one of the following conditions holds:

$\left(C_{1}\right)$ There exist $\rho_{1}, \rho_{2} \in(0, \infty), \frac{\rho_{1}}{c}<\rho_{2}$, such that $\left(I_{\rho_{1}}^{0}\right)$ and $\left(I_{\rho_{2}}^{1}\right)$ hold,

$\left(C_{2}\right)$ There exist $\rho_{1}, \rho_{2} \in(0, \infty), \rho_{1}<\rho_{2}$, such that $\left(I_{\rho_{1}}^{1}\right)$ and $\left(I_{\rho_{2}}^{0}\right)$ hold, then (1) has at least one nontrivial solution in $K$.

If one of the following conditions holds:

$\left(C_{3}\right)$ There exist $\rho_{1}, \rho_{2}, \rho_{3} \in(0, \infty), \frac{\rho_{1}}{c}<\rho_{2}<\rho_{3}$, such that $\left(I_{\rho_{1}}^{0}\right),\left(I_{\rho_{2}}^{1}\right)$, and $\left(I_{\rho_{3}}^{0}\right)$ hold,

$\left(C_{4}\right)$ There exist $\rho_{1}, \rho_{2}, \rho_{3} \in(0, \infty)$, with $\rho_{1}<\rho_{2}$ and $\frac{\rho_{2}}{c}<\rho_{3}$, such that $\left(I_{\rho_{1}}^{1}\right),\left(I_{\rho_{2}}^{0}\right)$, and $\left(I_{\rho_{3}}^{1}\right)$ hold,

then (1) has at least two nontrivial solutions in $K$.

Analogous results can be obtained to prove the existence of more than two solutions.

\section{Examples}

Example 7 Consider the following boundary value problem:

$$
\left\{\begin{array}{l}
u^{(3)}(t)=\lambda \frac{e^{t}\left(|u(t)|+\left|u^{\prime}(t)\right|+\left|u^{\prime \prime}(t)\right|\right)}{1+(u(t))^{2}}, \quad t \in[0,1], \\
u(0)=-u(1), \quad u^{\prime}(0)=\frac{1}{2} u^{\prime}(1), \quad u^{\prime \prime}(0)=0 .
\end{array}\right.
$$

Solutions of (4) correspond to the fixed points of the following operator:

$$
\mathcal{T} u(t)=\lambda \int_{0}^{1} G(t, s) f\left(s, u(s), u^{\prime}(s), u^{\prime \prime}(s)\right) \mathrm{d} s, \quad t \in[0,1],
$$

where the Green's function $G$ has the following expression:

$$
G(t, s)=\frac{1}{4} \begin{cases}(1-s)(-3+s+4 t), & t \leq s \\ (-3+s(s+4)+2 t(t+2)-8 s t), & s<t\end{cases}
$$


We note that this integral operator is a particular case of (1) for $T=1, m=2, k \equiv G$, and $f(t, x, y, z)=\frac{\left.e^{t}|| x|+| y|+| z \mid\right)}{1+x^{2}}$. We will check now that the kernel $G$ satisfies conditions $\left(H_{1}\right)-$ $\left(H_{5}\right)$. To do that, we need to calculate the explicit expression of the first and second partial derivatives of $G$,

$$
\begin{aligned}
& \frac{\partial G}{\partial t}(t, s)= \begin{cases}1-s, & t \leq s, \\
1-2 s+t, & s<t,\end{cases} \\
& \frac{\partial^{2} G}{\partial t^{2}}(t, s)= \begin{cases}0, & t<s, \\
1, & s<t .\end{cases}
\end{aligned}
$$

Using this expressions, we are able to check that the required conditions hold:

$\left(H_{1}\right)$ Let $\tau \in I$ be fixed. Both $G$ and $\frac{\partial G}{\partial t}$ are uniformly continuous, so the hypothesis is immediate for $i=0,1$. Moreover, for $\frac{\partial^{2} G}{\partial t^{2}}$ (that is, $i=m=2$ ), we can take $Z_{\tau}=\{\tau\}$, and we have that

$$
\left|\frac{\partial^{2} G}{\partial t^{2}}(t, s)-\frac{\partial^{2} G}{\partial t^{2}}(\tau, s)\right|=|1-1|=0, \quad \forall s<\min \{t, \tau\}
$$

and

$$
\left|\frac{\partial^{2} G}{\partial t^{2}}(t, s)-\frac{\partial^{2} G}{\partial t^{2}}(\tau, s)\right|=|0-0|=0, \quad \forall s>\max \{t, \tau\}
$$

so the hypothesis holds.

$\left(H_{2}\right)$ It occurs that $G(t, s) \geq 0$ for all $t \in\left[t_{0}, 1\right], s \in[0,1]$, with $t_{0} \approx 0.6133$. Thus, $\left[m_{0}, n_{0}\right]=$ $\left[t_{0}, 1\right]$.

Moreover, both $\frac{\partial G}{\partial t}$ and $\frac{\partial^{2} G}{\partial t^{2}}$ are nonnegative on the square $[0,1] \times[0,1]$, which means that $\left[m_{1}, n_{1}\right]=\left[m_{2}, n_{2}\right]=[0,1]$.

$\left(H_{3}\right)$ It can be checked that

$$
|G(t, s)| \leq \frac{1}{4}\left(3-4 s+s^{2}\right), \quad \text { for all } t \in[0,1], s \in[0,1],
$$

and the equality holds for $t=0$ and $t=1$, so the choice $h_{0}(s)=\frac{1}{4}\left(3-4 s+s^{2}\right)$ is optimal. This inequality can be easily proved by taking into account that, since $\frac{\partial G}{\partial t}$ is nonnegative, then $G(\cdot, s)$ is nondecreasing for every $s \in[0,1]$ and, therefore,

$$
|G(t, s)| \leq \max \{|G(0, s)|,|G(1, s)|\}=\frac{1}{4}\left(3-4 s+s^{2}\right) .
$$

For the first derivative, it holds that

$$
\left|\frac{\partial G}{\partial t}(t, s)\right| \leq 2(1-s) \quad \text { for all } t \in[0,1], s \in[0,1]
$$

and the equality holds for $t=1$, so $h_{1}(s)=2(1-s)$ is also optimal. 
Finally,

$$
\left|\frac{\partial^{2} G}{\partial t^{2}}(t, s)\right| \leq 1 \quad \text { for } t \in[0,1] \text { and a.e. } s \in[0,1]
$$

and $h_{2}(s)=1$ is trivially optimal.

$\left(H_{4}\right)$ If we take $\phi_{0}(s)=h_{0}(s)=\frac{1}{4}\left(3-4 s+s^{2}\right),\left[c_{0}, d_{0}\right]=[0,1]$, and $\left[a_{0}, b_{0}\right]=\left[t_{1}, 1\right]$ with $t_{1}>t_{0}\left(t_{0}\right.$ given in $\left.\left(H_{2}\right)\right)$, it holds that there exists a constant $\xi_{0}\left(t_{1}\right) \in(0,1)$ such that

$$
G(t, s) \geq \xi_{0}\left(t_{1}\right) \phi_{0}(s) \quad \text { for all } t \in\left[t_{1}, 1\right], s \in[0,1]
$$

We note that the bigger $t_{1}$ is, the bigger the constant $\xi_{0}\left(t_{1}\right)$ is. For instance, if we take $t_{1}=0.62$, we can choose $\xi_{0}=\frac{1}{75}$.

With regard to the first derivative of $G$, it satisfies that

$$
\frac{\partial G}{\partial t}(t, s) \leq 2(1-s) \quad \text { for all } t \in[0,1], s \in[0,1]
$$

that is, we could take $\phi_{1}(s)=h_{1}(s)=2(1-s),\left[c_{1}, d_{1}\right]=[0,1], \xi_{1}=\frac{1}{2}$, and $\left[a_{1}, b_{1}\right]=$ $[0,1]$.

Finally, for the second derivative of $G$, there do not exist a suitable function $\phi_{2}$ and a constant $\xi_{2}$ for which the inequalities in $\left(H_{4}\right)$ hold.

As a consequence, we deduce that $J_{1}=\{0,1\}$.

Moreover, it is obvious that $\int_{a_{i}}^{b_{i}} \phi_{i}(s) \mathrm{d} s>0$ for $i=0,1$.

$\left(H_{5}\right)$ It is immediately deduced from the proofs of the previous conditions.

Moreover, the nonlinearity $f$ satisfies condition $\left(H_{6}\right)$.

We will work in the cone

$$
\begin{aligned}
K= & \left\{u \in \mathcal{C}^{2}([0,1], \mathbb{R}): u(t) \geq 0, t \in\left[t_{0}, 1\right], u^{\prime}(t), u^{\prime \prime}(t) \geq 0, t \in[0,1] ;\right. \\
& \left.\min _{t \in\left[t_{1}, 1\right]} u(t) \geq \xi_{0}\left(t_{1}\right)\|u\|_{[0,1]}, \min _{t \in[0,1]} u^{\prime}(t) \geq \frac{1}{2}\left\|u^{\prime}\right\|_{[0,1]}\right\} .
\end{aligned}
$$

With the notation introduced in Sect. 3, we obtain the following values for the constants involved in Theorem 3:

$$
\Lambda^{0}=\frac{1}{3}, \quad \Lambda^{1}=1, \quad \Lambda^{2}=1,
$$

and therefore

$$
\begin{aligned}
& \bar{\Lambda}=3 \max \left\{\Lambda^{0}, \Lambda^{1}, \Lambda^{2}\right\}=3, \\
& \Lambda_{0}=\xi_{0}\left(t_{1}\right)\left(\frac{1}{3}-\frac{3}{4} t_{1}+\frac{1}{2} t_{1}^{2}-\frac{1}{12} t_{1}^{3}\right), \quad \Lambda_{1}=\frac{1}{2},
\end{aligned}
$$

and so

$$
\underline{\Lambda}=\max \left\{\xi_{0}^{2}\left(t_{1}\right)\left(\frac{1}{3}-\frac{3}{4} t_{1}+\frac{1}{2} t_{1}^{2}-\frac{1}{12} t_{1}^{3}\right), \frac{1}{4}\right\} .
$$


We note that, since $\xi_{0}\left(t_{1}\right) \in(0,1)$,

$$
\xi_{0}^{2}\left(t_{1}\right)\left(\frac{1}{3}-\frac{3}{4} t_{1}+\frac{1}{2} t_{1}^{2}-\frac{1}{12} t_{1}^{3}\right)<\frac{1}{3}-\frac{3}{4} t_{1}+\frac{1}{2} t_{1}^{2}-\frac{1}{12} t_{1}^{3}
$$

and it is easy to see that the right-hand side of previous inequality decreases with $t_{1}$ and, in particular, it is always smaller than $\frac{1}{4}$. Thus, $\underline{\Lambda}=\frac{1}{4}$, independently of the value of $t_{1}$.

On the other hand, we obtain the following values for the limits over the nonlinearity $f$ :

$$
\begin{aligned}
& f_{0}=\liminf _{|x|,|y|,|z| \rightarrow 0} \min _{t \in[0,1]} \frac{e^{t}(|x|+|y|+|z|)}{\left(1+x^{2}\right)(|x|+|y|+|z|)}=\lim _{|x|,|y|,|z| \rightarrow 0} \frac{1}{\left(1+x^{2}\right)}=1, \\
& f^{\infty}=\limsup _{|x|,|y|,|z| \rightarrow \infty} \max _{t \in[0,1]} \frac{e^{t}(|x|+|y|+|z|)}{\left(1+x^{2}\right)(|x|+|y|+|z|)}=\lim _{|x|,|y|,|z| \rightarrow \infty} \frac{e}{\left(1+x^{2}\right)}=0 .
\end{aligned}
$$

Therefore, from Theorem 3, we deduce that, for all $\lambda \in(4, \infty), \mathcal{T}$ has at least a fixed point in the cone $K$, with independence of the choice of $t_{1}$. This fixed point is a nontrivial solution of (4).

On the other hand, we will prove that it is not possible to apply Theorem 6 to this example. With the notation introduced in Lemma 5, we have that

$$
f_{\rho}^{0}=\inf \left\{\frac{e^{t}(|x|+|y|+|z|)}{\rho\left(x^{2}+1\right)}: t \in\left[t_{1}, 1\right], x \in\left[0, \frac{\rho}{\xi_{0}\left(t_{1}\right)}\right], y \in[0,2 \rho], z \in[0, \rho]\right\}=0
$$

and

$$
f_{\rho}^{1}=\inf \left\{\frac{e^{t}(|x|+|y|+|z|)}{\rho\left(x^{2}+1\right)}: t \in[0,1], x \in\left[0, \frac{\rho}{\xi_{0}\left(t_{1}\right)}\right], y \in[0,2 \rho], z \in[0, \rho]\right\}=0
$$

and therefore, there does not exist any $\rho$ such that condition $\left(I_{\rho}^{0}\right)$ holds. Thus Theorem 6 is not applicable to this example.

Example 8 Consider now the following Lidstone fourth order problem:

$$
\left\{\begin{array}{l}
u^{(4)}(t)=\lambda t\left(e^{u(t)}+\left(u^{\prime}(t)\right)^{2}+\left(u^{\prime \prime}(t)\right)^{2}+\left(u^{\prime \prime \prime}(t)\right)^{2}\right), \quad t \in[0,1] \\
u(0)=u(1)=u^{\prime \prime}(0)=u^{\prime \prime}(1)=0
\end{array}\right.
$$

Fourth order differential equations with these boundary conditions have been applied for studying the bending of simply supported elastic beams $[20,22]$ or suspension bridges [6, 19].

The solutions of problem (5) coincide with the fixed points of

$$
\mathcal{T} u(t)=\lambda \int_{0}^{1} G(t, s) f\left(s, u(s), u^{\prime}(s), u^{\prime \prime}(s), u^{\prime \prime \prime}(s)\right) \mathrm{d} s, \quad t \in[0,1]
$$

where $G$ has the following expression:

$$
G(t, s)=\frac{1}{6} \begin{cases}t(1-s)\left(2 s-s^{2}-t^{2}\right), & t \leq s \\ s(1-t)\left(2 t-t^{2}-s^{2}\right), & s<t\end{cases}
$$


Clearly, the previous operator is a particular case of (1) for $T=1, m=3, k \equiv G$, and $f(t, x, y, z, w)=t\left(e^{x}+y^{2}+z^{2}+w^{2}\right)$.

Next, we will give the explicit expressions of the first, second, and third derivatives of the Green's function:

$$
\begin{aligned}
& \frac{\partial G}{\partial t}(t, s)=\frac{1}{6} \begin{cases}-(1-s)\left(-2 s+s^{2}+3 t^{2}\right), & t \leq s, \\
s\left(2+s^{2}+3 t^{2}-6 t\right), & s<t,\end{cases} \\
& \frac{\partial^{2} G}{\partial t^{2}}(t, s)= \begin{cases}-t(1-s), & t \leq s, \\
-s(1-t), & s<t,\end{cases} \\
& \frac{\partial^{3} G}{\partial t^{3}}(t, s)= \begin{cases}-(1-s), & t<s, \\
s, & s<t,\end{cases}
\end{aligned}
$$

and now we will see that they satisfy the required hypotheses:

$\left(H_{1}\right)$ As in the previous example, it is easy to verify that this condition holds.

$\left(H_{2}\right)$ The Green's function $G$ is nonnegative on $[0,1] \times[0,1]$ (in fact, it is positive on $(0,1) \times(0,1))$. Therefore $\left[m_{0}, n_{0}\right]=[0,1]$.

For the first derivative, it holds that

$$
\frac{\partial G}{\partial t}(t, s) \geq 0 \quad \text { for all } t \in\left[0, t_{2}\right], s \in[0,1]
$$

with $t_{2}=1-\frac{\sqrt{3}}{3} \approx 0.42265$. Thus $\left[m_{1}, n_{1}\right]=\left[0, t_{2}\right]$.

With respect to the second derivative, it is immediate to see that it is nonpositive on its square of definition. However, it is zero on the boundary of the square, so we could take $\left[m_{2}, n_{2}\right]=\{0\}$ (it would also be possible to choose $\left[m_{2}, n_{2}\right]=\{1\}$ ).

Finally, the third derivative is nonnegative on the triangle $\{(t, s) \in[0,1] \times[0,1]$ : $s<t\}$, that is, $\left[m_{3}, n_{3}\right]=\{1\}$.

$\left(H_{3}\right)$ We have that

$$
|G(t, s)|=G(t, s) \leq h_{0}(s) \quad \text { for all } t \in[0,1], s \in[0,1]
$$

where

$$
h_{0}(s)=\frac{1}{9 \sqrt{3}} \begin{cases}s\left(1-s^{2}\right)^{\frac{3}{2}}, & 0 \leq s \leq \frac{1}{2} \\ (1-s)\left(2 s-s^{2}\right)^{\frac{3}{2}}, & \frac{1}{2}<s \leq 1\end{cases}
$$

The previous inequality has been proved in [24].

The previous inequality is optimal in the sense that, for each $s \in[0,1]$, there exists at least one value of $t \in[0,1]$ for which the equality is satisfied.

Analogously, it holds that

$$
\left|\frac{\partial G}{\partial t}(t, s)\right| \leq h_{1}(s) \quad \text { for all } t \in[0,1], s \in[0,1]
$$


for

$$
h_{1}(s)=\frac{1}{6} s(1-s) \begin{cases}2-s, & 0 \leq s \leq \frac{1}{2} \\ 1+s, & \frac{1}{2}<s \leq 1\end{cases}
$$

and the equality holds for $0 \leq s \leq \frac{1}{2}$ at $t=0$ and for $\frac{1}{2}<s \leq 1$ at $t=1$, so this choice of $h_{1}$ is optimal.

For the second derivative, we have that

$$
\left|\frac{\partial^{2} G}{\partial t^{2}}(t, s)\right| \leq s(1-s) \equiv h_{2}(s) \quad \text { for all } t \in[0,1], s \in[0,1]
$$

and the inequality is optimal in the same way as for the Green's function $G$.

With regard to the third derivative, it satisfies that

$$
\left|\frac{\partial^{3} G}{\partial t^{3}}(t, s)\right| \leq \max \{s, 1-s\} \equiv h_{3}(s) \quad \text { for } t \in[0,1] \text { and a.e. } s \in[0,1],
$$

and the inequality is also optimal.

$\left(H_{4}\right)$ If we choose $\phi_{0}(s)=h_{0}(s)$, given in $\left(H_{3}\right)$, and $\left[c_{0}, d_{0}\right]=[0,1]$, then for any closed interval $\left[a_{0}, b_{0}\right] \subset(0,1)$, it is possible to find a constant $\xi_{0}\left(a_{0}, b_{0}\right) \in(0,1)$ such that

$$
G(t, s) \geq \xi_{0}\left(a_{0}, b_{0}\right) \phi_{0}(s) \quad \text { for all } t \in\left[a_{0}, b_{0}\right], s \in[0,1] .
$$

This has been proved in [24] with an explicit function. Of course, it is satisfied that the bigger the interval $\left[a_{0}, b_{0}\right]$ is, the smaller $\xi_{0}\left(a_{0}, b_{0}\right)$ needs to be.

Analogously, we can take $\phi_{1}(s)=h_{1}(s)$ and $\left[c_{1}, d_{1}\right]=[0,1]$, and it holds that, for any interval $\left[0, b_{1}\right]$ with $b_{1}<1-\frac{\sqrt{3}}{3}$, there exists $\xi_{1}\left(b_{1}\right) \in(0,1)$ such that

$$
\frac{\partial G}{\partial t}(t, s) \geq \xi_{1}\left(b_{1}\right) \phi_{1}(s) \quad \text { for all } t \in\left[0, b_{1}\right], s \in[0,1] .
$$

Finally, with respect to the second derivative of the Green's function $G$, there does not exist any pair of function $\phi_{2}$ and constant $\xi_{2}$ such that the inequalities in $\left(H_{4}\right)$ hold. The same occurs with the third derivative of $G$. Therefore $J_{1}=\{0,1\}$.

$\left(H_{5}\right)$ It is a direct consequence of $\left(H_{2}\right)$.

Clearly, $f$ satisfies $\left(H_{6}\right)$ and $\left(H_{7}\right)$.

As a consequence of the properties of the Green's function that we have just seen, we will work in the cone

$$
\begin{aligned}
K= & \left\{u \in \mathcal{C}^{3}([0,1], \mathbb{R}): u(t) \geq 0, t \in[0,1], u^{\prime}(t) \geq 0, t \in\left[0, t_{2}\right],\right. \\
& u^{\prime \prime}(t) \geq 0, t \in\{0,1\}, u^{\prime \prime \prime}(1) \geq 0, \\
& \min _{t \in\left[a_{0}, b_{0}\right]} u(t) \geq \xi_{0}\left(a_{0}, b_{0}\right)\|u\|_{[0,1]}, \\
& \left.\min _{t \in\left[0, b_{1}\right]} u^{\prime}(t) \geq \xi_{1}\left(b_{1}\right)\left\|u^{\prime}\right\|_{[0,1]}\right\} .
\end{aligned}
$$

Moreover, we will make all the calculations with the values $\left[a_{0}, b_{0}\right]=[0.1,0.9], \xi_{0}=\frac{1}{4}$, $\left[0, b_{1}\right]=\left[0, \frac{1}{3}\right]$, and $\xi_{1}=\frac{1}{6}$. 
In this case, with the notation introduced in Lemma 4, we have that

$$
\frac{1}{N}=\max \left\{\frac{5}{384}, \frac{1}{24}, \frac{1}{8}, \frac{1}{2}\right\}=\frac{1}{2}
$$

and

$$
f^{\rho_{2}}=\sup \left\{\frac{t\left(e^{x}+y^{2}+z^{2}+w^{2}\right)}{\rho_{2}}: t \in[0,1], x, y, z, w \in\left[-\rho_{2}, \rho_{2}\right]\right\}=\frac{e^{\rho_{2}}+3 \rho_{2}^{2}}{\rho_{2}}
$$

and so $\left(I_{\rho_{2}}^{1}\right)$ holds for any $\lambda<\frac{2 \rho_{2}}{e^{\rho_{2}+3 \rho_{2}^{2}}}$.

Analogously, with the notation used in Lemma 5,

$$
\begin{aligned}
\frac{1}{M_{0}} & =\frac{29}{7500}, \quad \frac{1}{M_{1}}=\frac{7}{1944}, \\
f_{\rho_{1}}^{0} & =\inf \left\{\frac{t\left(e^{x}+y^{2}+z^{2}+w^{2}\right)}{\rho_{1}}: t \in[0.1,0.9], x \in\left[0,4 \rho_{1}\right], y \in\left[0,6 \rho_{1}\right], z, w \in\left[0, \rho_{1}\right]\right\} \\
& =\frac{0.1}{\rho_{1}}
\end{aligned}
$$

and

$$
f_{\rho}^{1}=\inf \left\{\frac{t\left(e^{x}+y^{2}+z^{2}+w^{2}\right)}{\rho_{1}}: t \in\left[0, \frac{1}{3}\right], x \in\left[0,4 \rho_{1}\right], y \in\left[0,6 \rho_{1}\right], z, w \in\left[0, \rho_{1}\right]\right\}=0
$$

and thus $\left(I_{\rho_{1}}^{0}\right)$ holds for $\lambda>\frac{75,000 \rho_{1}}{29}$.

Therefore, as a consequence of $\left(C_{1}\right)$ in Theorem 6 , for any pair of values $\rho_{1}, \rho_{2}>0$ such that $\rho_{1}<c \rho_{2}=\frac{\rho_{2}}{6}$ and

$$
\frac{75,000 \rho_{1}}{29}<\frac{2 \rho_{2}}{e^{\rho_{2}}+3 \rho_{2}^{2}}
$$

problem (5) has at least a nontrivial solution for all

$$
\lambda \in\left(\frac{75,000 \rho_{1}}{29}, \frac{2 \rho_{2}}{e^{\rho_{2}}+3 \rho_{2}^{2}}\right) .
$$

In particular, there exists at least a nontrivial solution of (5) for all $\lambda \in(0,0.4171)$.

On the other hand, we obtain that

$$
f_{0}=\liminf _{|x|,|y|,|z|,|w| \rightarrow 0} \min _{t \in[0,1]} \frac{t\left(e^{x}+y^{2}+z^{2}+w^{2}\right)}{|x|+|y|+|z|+|w|}=0,
$$

and thus Theorem 3 cannot be applied to this example.

\section{Application to some $2 n$-th order problems}

In this section we contribute to filling some gaps on the study of general $2 n$-th order Lidstone boundary value problems; for $n \geq 1$, usually the nonlinearities may depend only on the even derivatives (see, for example, $[5,18,23,26]$ ), or general complementary Lidstone 
problems (see [25] and the references therein). Therefore, we consider the following problem, with a full nonlinearity:

$$
\left\{\begin{array}{l}
u^{(2 n)}(t)=f\left(t, u(t), \ldots, u^{(2 n-1)}(t)\right), \quad t \in[0,1] \\
u^{(2 k)}(0)=u^{(2 k)}(1)=0, \quad k=0, \ldots, n-1
\end{array}\right.
$$

and denote by $G(t, s)$ the related Green's function.

It can be checked that, for $n \geq 2, g(t, s)=\frac{\partial^{2 n-4} G}{\partial t^{2 n-4}}(t, s)$ is the Green's function related to the problem

$$
\left\{\begin{array}{l}
u^{(4)}(t)=0, \quad t \in[0,1], \\
u(0)=u(1)=u^{\prime \prime}(0)=u^{\prime \prime}(1)=0,
\end{array}\right.
$$

whose explicit expression has been calculated in Example 8. As a consequence of the calculations made in that example, we know that the following facts hold for $n \geq 2$ :

- $\frac{\partial^{2 n-4} G}{\partial t^{2 n-4}}(t, s)=g(t, s) \geq 0$ on $[0,1] \times[0,1]$ and $\frac{\partial^{2 n-4} G}{\partial t^{2 n-4}}(t, s)=0$ on the boundary of the square.

- $\frac{\partial^{2 n-3} G}{\partial t^{2 n-3}}(t, s)=\frac{\partial g}{\partial t}(t, s) \geq 0$ on $\left[0, t_{2}\right] \times[0,1]$, with $t_{2}=1-\frac{\sqrt{3}}{3}$.

- $\frac{\partial^{2 n-2} G}{\partial t^{2 n-2}}(t, s)=\frac{\partial^{2} g}{\partial t^{2}}(t, s) \leq 0$ on $[0,1] \times[0,1]$, and $\frac{\partial^{2 n-2} G}{\partial t^{2 n-2}}(t, s)=0$ on the boundary of the square.

- $\frac{\partial^{2 n-1} G}{\partial t^{2 n-1}}(t, s)=\frac{\partial^{3} g}{\partial t^{3}}(1, s) \geq 0$ for $s \in[0,1]$.

With this information, we can obtain some results about the constant sign both of the derivatives of smaller order of $G$ and of the Green's function itself.

1. Since $\frac{\partial^{2 n-4} G}{\partial t^{2 n-4}}(t, s) \geq 0$, for $n \geq 3$, it holds that for each fixed $s \in[0,1], \frac{\partial^{2 n-5} G}{\partial t^{2 n-5}}(\cdot, s)$ is increasing.

Assume that it is nonnegative. Then it would occur that $\frac{\partial^{2 n-6} G}{\partial t^{2 n-6}}(\cdot, s)$ is also increasing and, since from the boundary value conditions it holds that $\frac{\partial^{2 n-6} G}{\partial t^{2 n-6}}(0, s)=\frac{\partial^{2 n-6} G}{\partial t^{2 n-6}}(1, s)=0$, we would conclude that $\frac{\partial^{2 n-6} G}{\partial t^{2 n-6}}(t, s)=0$ on $[0,1] \times[0,1]$, which is not possible.

The same argument holds if we assume that $\frac{\partial^{2 n-5} G}{\partial t^{2 n-5}}(\cdot, s)$ is nonpositive.

Therefore, necessarily $\frac{\partial^{2 n-5} G}{\partial t^{2 n-5}}(\cdot, s)$ is sign-changing and, since it is increasing, we know for sure that $\frac{\partial^{2 n-5} G}{\partial t^{2 n-5}}(0, s)<0$ and $\frac{\partial^{2 n-5} G}{\partial t^{2 n-5}}(1, s)>0$ for all $s \in[0,1]$.

2. Now, since $\frac{\partial^{2 n-5} G}{\partial t^{2 n-5}}(\cdot, s)$ is sign-changing and increasing, $\frac{\partial^{2 n-6} G}{\partial t^{2 n-6}}(\cdot, s)$ will be first decreasing and then increasing. This, together with the boundary value conditions $\frac{\partial^{2 n-6} G}{\partial t^{2 n-6}}(0, s)=\frac{\partial^{2 n-6} G}{\partial t^{2 n-6}}(1, s)=0$, implies that $\frac{\partial^{2 n-6} G}{\partial t^{2 n-6}}$ is nonpositive.

3. Since $\frac{\partial^{2 n-6} G}{\partial t^{2 n-6}}$ is nonpositive, we can follow an analogous argument to the one made in 1. to deduce that $\frac{\partial^{2 n-7} G}{\partial t^{2 n-7}}$ is sign-changing and decreasing. In particular, this implies that $\frac{\partial^{2 n-7} G}{\partial t^{2 n-7}}(0, s)>0$ and $\frac{\partial^{2 n-7} G}{\partial t^{2 n-7}}(1, s)<0$ for all $s \in[0,1]$ and $n \geq 4$.

4. Finally, analogously to 2 ., we can deduce that $\frac{\partial^{2 n-8} G}{\partial t^{2 n-8}}$ is nonnegative on $[0,1] \times[0,1]$ for $n \geq 4$.

We note that we could repeat all the previous arguments iteratively and this way we could deduce the following sign-criteria for the derivatives of $G$. So, for $n \geq \frac{k}{2}$ :

- If $k \equiv 0(\bmod 4)$, then $\frac{\partial^{2 n-k} G}{\partial t^{2 n-k}}(t, s) \geq 0$ on $[0,1] \times[0,1]$.

- If $k \equiv 1(\bmod 4)$, then $\frac{\partial^{2 n-k} G}{\partial t^{2 n-k}}(\cdot, s)$ is sign-changing and increasing for every $s \in[0,1]$. In particular, $\frac{\partial^{2 n-k} G}{\partial t^{2 n-k}}(0, s)<0$ and $\frac{\partial^{2 n-k} G}{\partial t^{2 n-k}}(1, s)>0$ for every $s \in[0,1]$. 
- If $k \equiv 2(\bmod 4)$, then $\frac{\partial^{2 n-k} G}{\partial t^{2 n-k}}(t, s) \leq 0$ on $[0,1] \times[0,1]$.

- If $k \equiv 3(\bmod 4)$, then $\frac{\partial^{2 n-k} G}{\partial t^{2 n-k}}(\cdot, s)$ is sign-changing and decreasing for every $s \in[0,1]$. In particular, $\frac{\partial^{2 n-k} G}{\partial t^{2 n-k}}(0, s)>0$ and $\frac{\partial^{2 n-k} G}{\partial t^{2 n-k}}(1, s)<0$ for every $s \in[0,1]$.

In particular, if $n$ is even, we could deduce that $G(t, s) \geq 0$ on $[0,1] \times[0,1]$ and, for $n$ odd, $G(t, s) \leq 0$ on $[0,1] \times[0,1]$.

Therefore, the Green's function and its derivatives satisfy the required hypotheses:

$\left(H_{1}\right)$ As in Example 8, this condition holds as a direct consequence of the general properties of the Green's function.

$\left(H_{2}\right)$ As we have just proved, we could take $\left[m_{2 n-i}, n_{2 n-i}\right]=[0,1]$ for $i \equiv 0(\bmod 4)$, $\left[m_{2 n-i}, n_{2 n-i}\right]=\{1\}$ for $i \equiv 1(\bmod 4),\left[m_{2 n-i}, n_{2 n-i}\right]=\{0\}$ for $i \equiv 2(\bmod 4)$, and $\left[m_{2 n-i}, n_{2 n-i}\right]=\{0\}$ for $i \equiv 3(\bmod 4)$.

$\left(H_{3}\right)$ It is enough to take $h_{i}(s)=\max \left\{\left|\frac{\partial^{i} G}{\partial t^{i}}(t, s)\right|: t \in[0,1]\right\}$ for $i \in J$.

$\left(H_{4}\right)$ For $n \geq 2$, we could take $J_{1}=\{2 n-4,2 n-3\}$. As a consequence of Example 8, we know that

$$
\left|\frac{\partial^{2 n-4} G}{\partial t^{2 n-4}}(t, s)\right|=\frac{\partial^{2 n-4} G}{\partial t^{2 n-4}}(t, s)=g(t, s) \leq \phi_{2 n-4}(s)
$$

with

$$
\phi_{2 n-4}(s)=\frac{1}{9 \sqrt{3}} \begin{cases}s\left(1-s^{2}\right)^{\frac{3}{2}}, & 0 \leq s \leq \frac{1}{2} \\ (1-s)\left(2 s-s^{2}\right)^{\frac{3}{2}}, & \frac{1}{2}<s \leq 1\end{cases}
$$

Moreover, it holds that, for any closed interval $\left[a_{2 n-4}, b_{2 n-4}\right] \subset[0,1]$, there exists a constant $\xi_{2 n-4}\left(a_{2 n-4}, b_{2 n-4}\right) \in(0,1)$ such that

$$
\frac{\partial^{2 n-4} G}{\partial t^{2 n-4}}(t, s) \geq \xi_{2 n-4}\left(a_{2 n-4}, b_{2 n-4}\right) \phi_{2 n-4}(s), \quad \text { for all } t \in\left[a_{2 n-4}, b_{2 n-4}\right], s \in[0,1] .
$$

Analogously, from Example 8 we know that

$$
\left|\frac{\partial^{2 n-3} G}{\partial t^{2 n-3}}(t, s)\right|=\left|\frac{\partial g}{\partial t}(t, s)\right| \leq \phi_{2 n-3}(s)=\frac{1}{6} s(1-s) \begin{cases}2-s, & 0 \leq s \leq \frac{1}{2} \\ 1+s, & \frac{1}{2}<s \leq 1\end{cases}
$$

for all $t \in[0,1], s \in[0,1]$ and for any interval $\left[0, b_{2 n-3}\right]$ with $b_{2 n-3}<1-\frac{\sqrt{3}}{3}$ there exists $\xi_{2 n-3}\left(b_{2 n_{3}}\right) \in(0,1)$ such that

$$
\frac{\partial^{2 n-3} G}{\partial t^{2 n-3}}(t, s) \geq \xi_{2 n-3}\left(b_{2 n_{3}}\right) \phi_{2 n-3}(s) \quad \text { for all } t \in\left[0, b_{2 n-3}\right], s \in[0,1] .
$$

$\left(H_{5}\right)$ As we have already seen, it holds that $\left[m_{2 n-4}, n_{2 n-4}\right]=[0,1]$. 
Then, we could work in the cone for $n \in \mathbb{N}$ such that $n \geq \max \left\{2, \frac{i}{2}\right\}$,

$$
\begin{aligned}
K= & \left\{u \in \mathcal{C}^{2 n-1}([0,1], \mathbb{R}): u^{(2 n-i)}(t) \geq 0, t \in[0,1], i \equiv 0 \quad \bmod 4 ;\right. \\
& u^{(2 n-i)}(1) \geq 0, i \equiv 1 \quad \bmod 4 ; \\
& u^{(2 n-i)}(0) \geq 0, i \equiv 2 \quad \bmod 4 ; \quad u^{(2 n-i)}(0) \geq 0, i \equiv 3 \quad \bmod 4 ; \\
& \min _{t \in\left[a_{2 n-4}, b_{2 n-4}\right]} u^{(2 n-4)}(t) \geq \xi_{2 n-4}\left(a_{2 n-4}, b_{2 n-4}\right)\left\|u^{(2 n-4)}\right\|_{[0,1]} ; \\
& \left.\min _{t \in\left[0, b_{2 n-3}\right]} u^{(2 n-3)}(t) \geq \xi_{2 n-3}\left(b_{2 n-3}\right)\left\|u^{(2 n-3)}\right\|_{[0,1]}\right\} .
\end{aligned}
$$

Thus, for any nonlinearity $f$ satisfying $\left(H_{6}\right)$ and either conditions of Theorem 3 or those of Theorem 6, it is possible to find nontrivial solution of problem (6).

\section{Acknowledgements}

This paper was mostly written during a stay of Lucía López-Somoza in Évora. Lucía López-Somoza would like to acknowledge her gratitude towards the Department of Mathematics of the University of Évora, and specially towards Professor Feliz Minhós for his kindness and hospitality.

\section{Funding}

First author was partially supported by Xunta de Galicia (Spain), project EM2014/032, AIE Spain and FEDER, grants MTM2013-43014-P, MTM2016-75140-P, and FPU scholarship, Ministerio de Educación, Cultura y Deporte, Spain. F. Minhós was supported by FCT-Fundação para a Ciência e a Tecnologia, via project UID/MAT/ 04674/2019.

\section{Availability of data and materials}

Data sharing not applicable to this article as no datasets were generated or analyzed during the current study.

\section{Competing interests}

There are no competing interests.

\section{Authors' contributions}

Both authors contributed equally to the writing of this paper. Both authors read and approved the final manuscript.

\section{Author details}

${ }^{1}$ Instituto de Matemáticas, Facultade de Matemáticas, Universidade de Santiago de Compostela, Santiago de Compostela, Spain. ${ }^{2}$ Departamento de Matemática, Escola de Ciências e Tecnologia, Universidade de Évora, Évora, Portugal. ${ }^{3}$ Centro de Investigação em Matemática e Aplicações (CIMA), Instituto de Investigação e Formação Avançada, Universidade de Évora, Évora, Portugal.

\section{Publisher's Note}

Springer Nature remains neutral with regard to jurisdictional claims in published maps and institutional affiliations.

Received: 1 June 2019 Accepted: 30 September 2019 Published online: 07 October 2019

\section{References}

1. Agarwal, R.P., O'Regan, D., Wong, P.J.Y.: Eigenvalues of a system of Fredholm integral equations. Math. Comput. Model. 39, 1113-1150 (2004)

2. Benmezai, A., Graef, J.R., Kong, L.: Positive solutions for abstract Hammerstein equations and applications. Commun. Math. Anal. 16, 47-65 (2014)

3. Cabada, A., Infante, G., Tojo, F.A.F.: Nontrivial solutions of Hammerstein integral equations with reflections. Bound. Value Probl. 2013, 86, 22 pp (2013)

4. Cabada, A., López-Somoza, L., Minhós, F.: Existence, non-existence and multiplicity results for a third order eigenvalue three-point boundary value problem. J. Nonlinear Sci. Appl. 10, 5445-5463 (2017)

5. Davis, J., Henderson, J., Wong, P.: General Lidstone problems: multiplicity and symmetry of solutions. J. Math. Anal. Appl. 251, 527-548 (2000)

6. Drábek, P., Holubová, G., Matas, A., Nečesal, P.: Nonlinear models of suspension bridges: discussion of results. Appl. Math. 48, 497-514 (2003)

7. Franco, D., Infante, G., O'Regan, D.: Nontrivial solutions in abstract cones for Hammerstein integral systems. Dyn. Contin. Discrete Impuls. Syst., Ser. A Math. Anal. 14, 837-850 (2007)

8. Graef, J., Kong, L., Minhós, F.: Generalized Hammerstein equations and applications. Results Math. 72, 369-383 (2017)

9. Granas, A., Dugundji, J.: Fixed Point Theory. Springer, Berlin (2003)

10. Guo, D., Lakshmikantham, V.: Nonlinear Problems in Abstract Cones. Academic Press, San Diego (1988) 
11. Guo, L., Sun, J., Zhao, Y.: Existence of positive solutions for nonlinear third-order three-point boundary value problems. Nonlinear Anal. 68, 3151-3158 (2008)

12. Hammerstein, A.: Nichtlineare Integralgleichungen nebst Anwendungen. Acta Math. 54, 117-176 (1930)

13. Infante, G., Minhós, F.: Nontrivial solutions of systems of Hammerstein integral equations with first derivative dependence. Mediterr. J. Math. 14, 242, 18 pp (2017)

14. Infante, G., Pietramala, P., Tojo, F.A.F.: Nontrivial solutions of local and nonlocal Neumann boundary-value problems. Proc. R. Soc. Edinb. 146A, 337-369 (2016)

15. Infante, G., Webb, J.R.L.: Nonzero solutions of Hammerstein integral equations with discontinuous kernels. J. Math. Anal. Appl. 272, 30-42 (2002)

16. Infante, G., Webb, J.R.L.: Three point boundary value problems with solutions that change sign. J. Integral Equ. Appl. 15, 37-57 (2003)

17. Jiang, C., Zhai, C.: Existence of nontrivial solutions for a nonlinear fourth-order boundary value problem via iterative method. J. Nonlinear Sci. Appl. 9, 4295-4304 (2016)

18. Jurkiewicz, M.: Existence result for the Lidstone boundary value problem at resonance. J. Math. Anal. Appl. 394, 248-259 (2012)

19. Lazer, A.C., McKenna, P.J.: Large-amplitude periodic oscillations in suspension bridges: some new connections with nonlinear analysis. SIAM Rev. 32, 537-578 (1990)

20. Minhós, F., Gyulov, T., Santos, A.I.: Lower and upper solutions for a fully nonlinear beam equations. Nonlinear Anal. 71, 281-292 (2009)

21. Minhós, F., Sousa, R.: On the solvability of third-order three point systems of differential equations with dependence on the first derivative. Bull. Braz. Math. Soc. 48(3), 485-503 (2016)

22. Timoshenko, S.P.: Theory of Elastic Stability. McGraw-Hill, New York (1961)

23. Wang, Y.: On 2nth-order Lidstone boundary value problems. J. Math. Anal. Appl. 312, 383-400 (2005)

24. Webb, J.R.L., Infante, G., Franco, D.: Positive solutions of nonlinear fourth-order boundary-value problems with local and non-local boundary conditions. Proc. R. Soc. Edinb. A 138(2), 427-446 (2008)

25. Wong, P.: Triple solutions of complementary Lidstone boundary value problems via fixed point theorems. Bound Value Probl. 2014, 125 (2014)

26. Zhang, B., Liu, X.: Existence of multiple symmetric positive solutions of higher order Lidstone problems. J. Math. Anal. Appl. 284, 672-689 (2003)

\section{Submit your manuscript to a SpringerOpen ${ }^{\circ}$ journal and benefit from:}

- Convenient online submission

- Rigorous peer review

- Open access: articles freely available online

- High visibility within the field

- Retaining the copyright to your article

Submit your next manuscript at $>$ springeropen.com 